\title{
Caractéristique Physico-Chimique Etdynamique des Formes Environnementales des Coccidies Entériques Dans Les Eaux De Sources, Puits et Cours D'eau dans La Commune d'Akono(Cameroun, Afrique Centrale)
}

\author{
Rose Pulcherie Ngakomo Ananga,
}

Laboratoire d'Hydrobiologie et Environnement

Université de Yaoundé, Cameroun

Gideon Aghaindum Ajeagah, Zeinab Abou Elnaga,

Laboratoire de Zoologie de l'université de Mansoura, Egypte, Laboratoire d'Hydrobiologie et Environnement Université de Yaoundé, Cameroun

\section{Pierre Ngassam,}

Laboratoire d'Hydrobiologie et Environnement

Université de Yaoundé, Cameroun

URL:http://dx.doi.org/10.19044/esj.2020.v16n3p161

\section{Resume}

L'objectif de cette étude vise à évaluer la qualité physico-chimique et parasitologique des eaux de sources, des puits et du cours d'eau dans la commune d'Akono. L'étude entreprise d'Avril 2017 à Mars 2018 a permis d'effectuer les échantillonnages pour des analyses physico-chimiques et parasitologiques. Les paramètres physiques tels que la température $\left(\mathrm{T}^{\circ} \mathrm{C}\right)$, la turbidité, les matières en suspension (MES) et la couleur et quelques paramètres chimiques : le $\mathrm{pH}$, la conductivité, l'oxygène, l'oxydabilité, les nitrates, les nitrites, l'azote ammoniacal ont été mesurés. Les eaux de la commune d'Akono sont légèrement acides $(\mathrm{Ph}=6,1 \pm 0,3 \mathrm{UC})$ et faiblement minéralisées (conductivité $=95,5 \pm 67,5 \mu \mathrm{S} / \mathrm{Cm}$ ). Les teneurs en azote obtenues sont inférieures aux normes. De fortes corrélations ont été mises en évidence entre la conductivité, la turbidité, la couleur, les MES et les densités des oocystes obtenues. Les analyses parasitologiques des échantillons d'eau ont permis d'identifier des oocystes du genre Cryptosporidium sp (141 oocystes/L), de Cyclospora cayetanensis (52 oocystes/L) et d'Isospora belli (40 oocystes/L). Les eaux ont été soumises à une pollution d'origine fécale dans la commune d'Akono. L'usage de ces eaux pourrait présenter un risque sanitaire pour les populations. 
Mots clés : Akono, Oocyste, Puits, Sources, Cours D’eau.

\title{
Physico-Chemical Characteristics and Dynamics of the Environmental Form of Intestinal Coccidies in the Springs, Wells and Streams of the Akono Municipality (Cameroon, Central Africa)
}

\author{
Rose Pulcherie Ngakomo Ananga, \\ Laboratoire d'Hydrobiologie et Environnement \\ Université de Yaoundé, Cameroun \\ Gideon Aghaindum Ajeagah, \\ Zeinab Abou Elnaga, \\ Laboratoire de Zoologie de l'université de Mansoura, Egypte, Laboratoire \\ d'Hydrobiologie et Environnement Université de Yaoundé, Cameroun
}

Pierre Ngassam,

Laboratoire d'Hydrobiologie et Environnement

Université de Yaoundé, Cameroun

\begin{abstract}
The objective of this study is intended to assess the physico-chemical quality and the parasitological quality of spring waters, wells and stream in the Akono city council. The study was carried out from April 2017 to March 2018. Within this time, samples for physicochemical and Parasitological analyses where collected. Physical parameters such as temperature $\left(\mathrm{T}{ }^{\circ} \mathrm{C}\right)$, turbidity, suspended solids (SS) and the color and some chemical parameters: $\mathrm{pH}$, conductivity, oxygen, the oxidability, nitrates, nitrites, ammonia were measured. The waters of Akono municipality are slightly acidic with an average $\mathrm{pH}$ of $6.1 \pm 0.3 \mathrm{UC}$. These waters are lowly mineralized with an average electrical conductivity of $95.5 \pm 67.5 \mu \mathrm{S} / \mathrm{cm}$. The concentrations of nitrogenous compounds obtained are slightly below substandard. Strong correlations were observed between electrical conductivity, turbidity, color, SS by the oocysts and densities obtained. Parasitological analyses were made by observation using an optical microscope $40 \mathrm{X}$ objective. The results reveal the presence of oocysts of Cryptosporidium sp. (141oocysts/L), Cyclospora cayetanensis $(5200 c y s t s / \mathrm{L})$ and Isospora belli (40oocysts/L). The water was subjected to pollution of faecal origin in the Akono municipality. The use of this waters could present a health risk for the population.
\end{abstract}


Keywords: Akono Municipality, Oocyst, Wells, Springs, Streams

\section{Introduction}

L'eau est une ressource indispensable à toute forme de vie sur la planète (Santos et al, 2010). En effet, cette ressource tient une place importante dans la vie de l'homme, qui l'utilise pour ses besoins alimentaires, ainsi que pour ses activités agricoles et industrielles. Cependant, elle demeure une ressource épuisable et fragile, car vulnérable à diverses types de pollutions (Vilagines, 2003). En outre, elle peut être source de maladies. A l'échelle mondiale, près de $90 \%$ des maladies diarrhéiques sont imputables à la mauvaise qualité de l'eau de boisson et à un assainissement insuffisant des eaux usées. Selon l'Organisation Mondiale de la Santé, plus de 2 millions de personnes, surtout des enfants de moins de cinq ans des pays en voie de développement meurent chaque année des maladies diarrhéiques du fait de l'insuffisance des mesures d'hygiènes et d'assainissement (OMS, 2014). La pollution et la rareté de l'eau sont en réalité un problème mondial dont les aspects et la portée sont différents selon les niveaux de développement des Nations. Au Cameroun, le volume d'eau produit ne couvre pas les besoins des populations. Ce déficit est plus marqué en milieu rural environ $86 \%$ des populations rurales n'ont pas accès à l'eau potable (INS, 2013). La commune d'Akono fait partie des communes rurales non desservie. Cette situation contraint ces populations à recourir aux eaux de sources, de puits, de forages et même de surface malgré les risques encourus. En effet, des études réalisées sur les eaux souterraines et de surfaces en zone urbaines et périurbaines ont montrées que ces eaux sont de mauvaise qualité et hébergent des germes témoins de contamination (Ajeagah et al., 2010 ; Tuekam Kayo, 2013 ; Kapso tchouankep, 2018).

La présente étude vise donc à évaluer la qualité physico-chimique et la dynamique des formes de résistance des coccidies entériques dans les eaux souterraines et de surface provenant de la commune d'Akono. Spécifiquement, il s'agira de: déterminer les paramètres physico-chimiques des points d'eau; d'isoler, d'identifier et de d'évaluer la densité des formes de résistances des coccidies présent dans les échantillons d'eau et évaluer l'influence des paramètres physico-chimiques sur la fluctuation des densités des oocystes de coccidies identifiés.

\section{Matériels et Méthodes}

\subsection{Site d'étude}

Akono est un arrondissement du département de la Mefou et Akono, qui se trouve dans la Province du Centre, au Sud de la capitale du Cameroun, Yaoundé. Cet arrondissement est Situé dans la zone équatoriale humide, entre 
$3^{\circ} 29^{\prime} 59^{\prime \prime}$ et $3^{\circ} 30^{\prime} 30^{\prime \prime}$ de latitude Nord et entre $11^{\circ} 19^{\prime} 36^{\prime \prime}$ et $11^{\circ} 20^{\prime}$ de longitude Est. Les moyennes de températures sont comprises entre 22 et $26^{\circ} \mathrm{C}$. Le climat comprend quatre saisons: une grande saison sèche (de Novembre à mi-Mars) ; une petite saison pluvieuse (de mi-Mars à mi-Juin) ; une petite saison sèche (de mi-Juin à mi-Août) et une grande saison pluvieuse (de miAoût à fin Octobre). Le territoire communal d'Akono a une superficie de 320 $\mathrm{Km}^{2}$. Le sol est de nature argileuse mais on y trouve par endroit la présence de la latérite ferralitique. Les activités les plus pratiquées sont le cacao culture, la caféiculture, l'artisanat avec l'exploitation du rotin et des bois de la forêt, l'élevage de la volaille (poule, perdrix...) et du bétail (porc, bœuf, mouton...). En plus, les populations exercent le commerce. Mais il y'a beaucoup de manquements : la population n'a pas accès à un meilleur système d'assainissement (Kamgho, 2011). la dominance des ouvrages d'assainissement autonomes (latrines), favorise la diffusion interne des agents pathogènes à travers la porosité du sol (Wéthé et al., 2003) et contribue à la pollution massive de la nappe phréatique. En outre l'absence du réseau de distribution d'eau potable entraine un accès difficile à l'eau potable ce qui favorise le développement des points d'eau d'approvisionnement comme les puits, les sources et les cours d'eau. D'autre part, cette rareté de la ressource hydrique de bonne qualité, peut être responsable de l'apparition des maladies diarrhéiques.

Le tableau I présente les coordonnées géographiques et caractéristiques des stations étudiées. Le choix des stations d'échantillonnage s'est faites suivant les critères tels que: les sources de contamination, l'usage de l'eau, l'importance du point d'eau pour les populations, le manque d'assainissement adéquat, l'hygiène précaire et l'accessibilité. Au total 06 stations ont été choisies à raison de 02 puits ( $\mathrm{P} 1$ et $\mathrm{P} 2), 02$ sources ( $\mathrm{S} 1$ et $\mathrm{S} 2)$, et 02 stations sur le cours d'eau Akono ( $\mathrm{C} 1$ et $\mathrm{C} 2$ ).

Tableau I : Coordonnées géographiques et caractéristiques des milieux aquatiques étudiés dans la localitéd'Akono

\begin{tabular}{|c|c|c|c|c|c|}
\hline \multirow{2}{*}{$\begin{array}{l}\text { Localité et } \\
\text { milieux } \\
\text { aquatiques }\end{array}$} & \multirow[b]{2}{*}{ Stations } & \multicolumn{3}{|c|}{ Coordonnés GPS } & \multirow{2}{*}{ Activités et caractéristiques } \\
\hline & & $\begin{array}{l}\text { Latitude } \\
\left({ }^{\circ} \mathrm{N}\right)\end{array}$ & $\begin{array}{l}\text { Longitude } \\
\left({ }^{\circ} \mathrm{E}\right)\end{array}$ & $\begin{array}{l}\text { Altitude } \\
\text { (m) }\end{array}$ & \\
\hline Cours d'eau & $\begin{array}{l}\text { C1 (situé en } \\
\text { amont du } \\
\text { cours d'eau) }\end{array}$ & $03^{\circ} 30^{\prime} 24,7^{\prime \prime}$ & $11^{\circ} 19^{\prime} 30^{\prime \prime}$ & 629 & $\begin{array}{l}\text { Le lit du cours à été modifié, les } \\
\text { berges sont recouvertes de } \\
\text { ciment et entouré de hautes } \\
\text { herbes }\end{array}$ \\
\hline Cours d'eau & $\begin{array}{l}\text { C2 (situé en } \\
\text { aval du } \\
\text { cours d'eau) }\end{array}$ & $3^{\circ} 30^{\prime} 21,8^{\prime \prime}$ & $11^{\circ} 19^{\prime} 27,7^{\prime}$, & 628 & $\begin{array}{l}\text { lessive, la baignade, laverie } \\
\text { automobile et abreuvage du } \\
\text { bétail }\end{array}$ \\
\hline \multirow[t]{2}{*}{ sources } & S1 & $3^{\circ} 29^{\prime} 55,5^{\prime \prime}$ & $11^{\circ} 19^{\prime} 46,8^{\prime \prime}$ & 661 & \multirow{2}{*}{$\begin{array}{l}\text { Sans couverture, infiltration des } \\
\text { eaux usées } \\
\text { agriculture }\end{array}$} \\
\hline & S2 & $3^{\circ} 29^{\prime} 51,6^{\prime \prime}$ & $11^{\circ} 19^{\prime \prime} 43,5^{\prime \prime}$ & 660 & \\
\hline
\end{tabular}




\begin{tabular}{|l|l|l|l|l|l|}
\hline & & & & & \\
\hline Puits & P1 & $3^{\circ} 30^{\prime} 5,7^{\prime}{ }^{\prime}$ & $11^{\circ} 19^{\prime} 40,1^{\prime}{ }^{\prime}$ & 678 & Margelle cimenté et couvercle \\
\cline { 2 - 6 } & P2 & $3^{\circ} 30^{\prime} 0,5^{\prime}$, & $1^{\circ} 19^{\prime} 54,8^{\prime}$, & 687 & $\begin{array}{l}\text { Absence de margelle, sans } \\
\text { couverture } \\
\text { Infiltration des eaux usées } \\
\text { Proximité avec les latrines }\end{array}$ \\
\hline
\end{tabular}

\subsection{Paramètres physicochimiques}

Les analyses physicochimiques se sont déroulées à la fois sur le terrain et au laboratoire (APHA, 1998 ; Rodier, 2009). Pour les analyses effectuées au laboratoire, les échantillons d'eau ont été prélevés sur le terrain à l'aide des flacons en polyéthylène à double bouchage de 250 et $1000 \mathrm{ml}$.

\subsubsection{Température de l'eau}

La température de l'eau à chaque station a été mesurée sur place à l'aide d'un thermomètre à mercure gradué au $1 / 10^{\circ} \mathrm{C}$.

2.3.2. Matières en Suspension (MES), couleur, turbidité, Nitrates et Nitrites

Les MES, la couleur, la turbidité, les Nitrates et les Nitrites ont été mesurées au laboratoire au spectrophotomètre HACH DR/3900.

\subsubsection{Potentiel d'Hydrogène $(\mathrm{pH})$, Conductivité électrique}

Les mesures de la Conductivité, du $\mathrm{pH}$, salinité ont été faites sur le terrain à l'aide d'un multi paramètres portatif de marque HANNA model HI 9829 de précision 0.01 .

\subsubsection{Oxygène dissous et les Matières organiques : Oxydabilité}

La teneur en oxygène dissous $\left(\mathrm{O}_{2}\right)$ a été mesuré au laboratoire après fixation sur le terrain. Et l'oxydabilité ou teneur en matière organique dégradable a été mesurée au laboratoire par la méthode volumétrique.

\subsection{Mesure des variables biologiques}

\subsubsection{Echantillonnage pour la recherche des oocystes}

Pour les analyses biologiques, les échantillons d'eau prélevés sont ramenés au laboratoire dans une enceinte réfrigérée et sont laissés pour sédimentation pendant 24 heures à température ambiante. Les dépôts obtenus ont ensuite été quantifiés et homogénéisés.

\subsubsection{Techniques d'identification}

Deux méthodes d'identification ont été utilisées pour isoler et dénombrer les oocystes. Il s'agit de la méthode de concentration et de la technique de Ziehl-Neelsen modifiée (Ajeagah et al., 2010 ). 


\subsubsection{Méthode de concentration}

La méthode de concentration a consisté à prélever un sous spécimen de $10 \mathrm{~mL}$ des dépôts obtenus par échantillon et distribuer dans les tubes à essai auxquels $0,5 \mathrm{~mL}$ de formol sont additionné. Ensuite après ajout de $5 \mathrm{~mL}$ d'eau distillée ainsi que quelques gouttes de colorant (Lugol, MIF, fuschine basique, hématoxyline ou le bleu de méthylène), qui colore les structures internes des oocystes.

Les contenus des tubes sont centrifugés à 500 tours/min pendant cinq minutes, pour favoriser la sédimentation des oocystes au fond du tube.

Le surnageant est éliminé en renversant doucement le tube, et, le culot est prélevé à l'aide d'une micropipette et posé sur des lames porte-objet. L'examen et le dénombrement des oocystes sont réalisés au microscope Olympus CK2 à l'objectif 40X et 100X

\subsubsection{Technique de Zielh-Neelsen modifiée}

La technique de Ziehl-Neelsen quant à elle a consisté à prélever $10 \mathrm{~mL}$ des dépôts obtenus qu'on introduit dans un tube à essai, puis ajouter une solution de sulfate de zinc (10\%) (pour favoriser la flottaison des parasites) au culot de $10 \mathrm{~mL}$, quiest ensuite centrifugé à 500 tours/min pendant cinq minutes. Le surnageant contenant les oocystes est prélevé à l'aide d'une micropipette et posé sur des lames porte-objet qui sont ensuite séchées, après fixation au méthanol et coloration à la fuchsine basique, les lames sont rincées à l'eau et à l'acide sulfurique (2\%). Une contre-coloration au bleu de méthylène $(5 \%)$ est ensuite réalisée. Après rinçage à l'eau et séchage àl'air, l'examen et le dénombrement des oocystes sont réalisées au microscope Olympus CK2 aux grossissements 40X.

\subsection{Analyse des résultats et test statistiques}

Le volume intégral $(\mathrm{Vx})$ du culot a été enregistré. Après homogénéisation du culot, un volume précis $(\mathrm{Vy}=10 \mathrm{~mL})$ a été prélevé et introduit dans un tube à essai. Le culot final de chaque tube à essai est réparti sur des lames, puis le nombre d'oocystes est compté. Le nombre total d'oocystes dans l'échantillon est calculé en multipliant la valeur obtenue sur toutes les lames par la fraction $\mathrm{Vx} / \mathrm{Vy}$ et le résultat est enfin ramené au litre et le nombre d'oocyste est noté par litre (oocyste/L) (Ajeagah et al., 2010).Les données collectées ont été groupées par saison en utilisant le programme Microsoft Excel 2010 et traitées en utilisant le logiciel SPSS version 20.0. La corrélation de Spearman a permis de mettre en évidence le degré de corrélation entre les paramètres physico-chimique (la conductivité électrique, les MES, la couleur et la turbidité) et parasitologiques (la densité des oocystes de Cryptosporidium sp et de Cyclospora cayetanensis). 


\section{Résultats}

3.1. Variances physicochimiques des sources, puits et cours d'eau de la localité d'Akono

\subsection{Variables physiques}

Les valeurs des paramètres physiques obtenues sont présentées sur la figure 1. Les eaux de la commune d'Akono ont une température moyenne de $24,5 \pm 0,5^{\circ} \mathrm{C}$. Cette moyenne a oscillée entre un minimum de 23,8 et un maximum de $26^{\circ} \mathrm{C}$ (figure $1 \mathrm{~A}$ ). La turbidité des eaux varie d'une station à une autre, les valeurs obtenues oscillent entre 2 et 43 FTU, la turbidité est plus élevée dans le cours d'eau. Les eaux souterraines (S1, S2, P1 et P2) sont moins turbides. Les eaux de puits contiennent peu de particules en suspensions par rapport aux eaux de sources et du cours d'eau, les teneurs des MES sont restées faibles et présentent des variations d'une station à une autre, les analyses montre des valeurs qui évoluent entre 4 et $24 \mathrm{mg} / \mathrm{L}$ avec une moyenne de $10,7 \pm 5,1 \mathrm{mg} / \mathrm{L}$ (figure 1C).Dans les milieux aquatiques étudiés, les valeurs de la couleur présentent de fortes variations d'une station à une autre. Elles varient entre 22,2 et 267Pt-Co avec une moyenne de 99,6 \pm 76,2Pt-Co (figure 1D), les eaux sont faiblement colorées au niveau des puits, moins colorées dans les sources et plus colorées dans le cours d'eau.
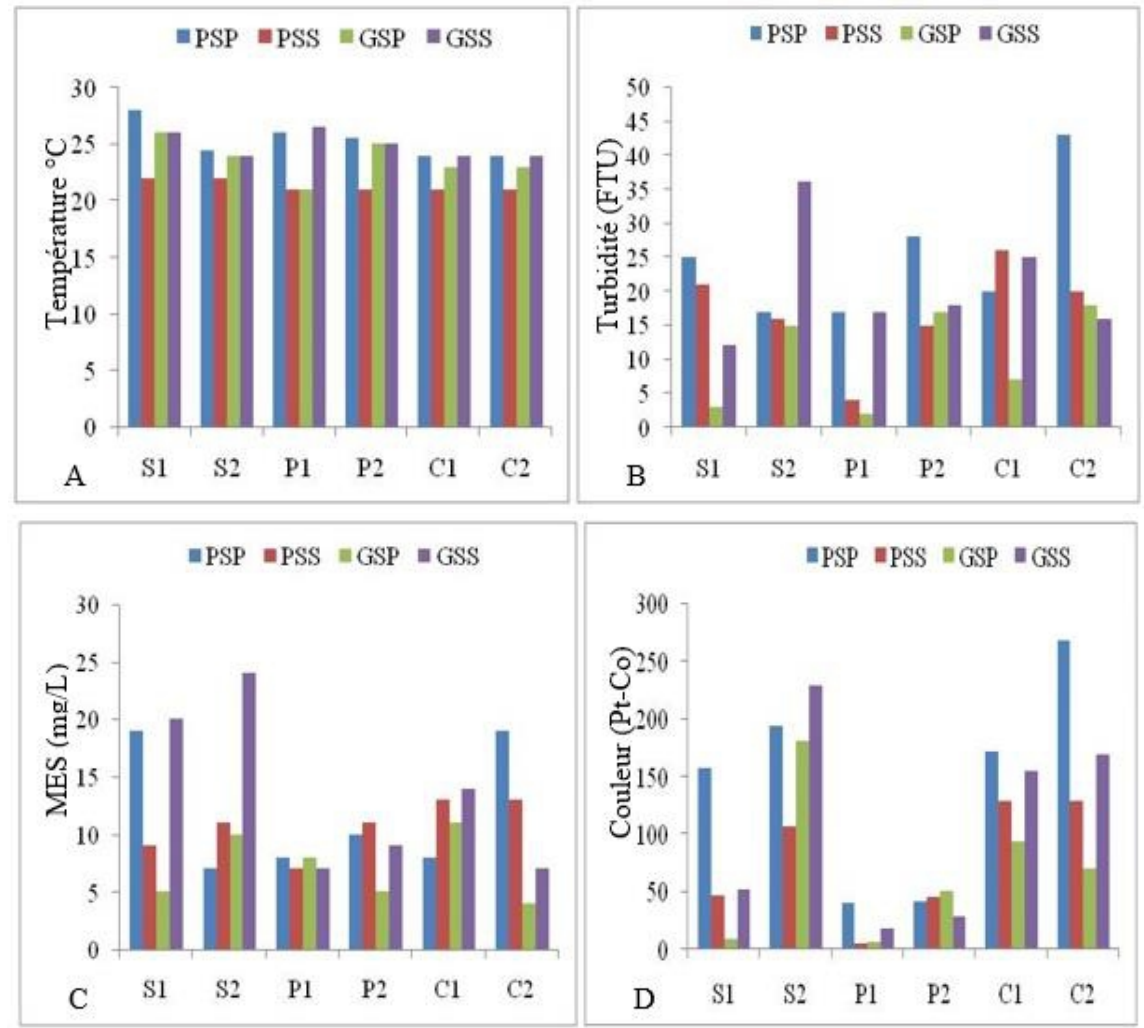

Figure 1 : variation des paramètres physiques dans les milieux aquatiques de la commune d'Akono : (A) température, (B) Turbidité, (C) MES et (D) couleur. 


\subsubsection{Variables chimiques}

les eaux de la commune d'Akono comme l'illustre la figure 3, ont un

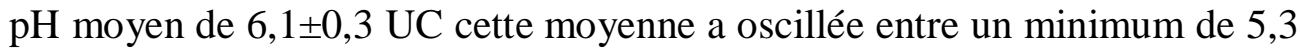
et maximum de 6,7 UC, révélant des eaux légèrement acide (figure $2 \mathrm{~A}$ et $2 \mathrm{~B}$ ). La conductivité électrique varie également d'une station à une autre. Les analyses montrent des valeurs plus élevée dans les eaux souterraines allant de 54 à $218 \mu \mathrm{S} / \mathrm{cm}$, avec une moyenne de $126,1 \pm 63,1 \mu \mathrm{S} / \mathrm{cm}$ par rapport aux eaux du cours d'eau, où elle est resté très faible pendant toute la période d'étude et varie entre 31 et $42 \mu \mathrm{S} / \mathrm{Cm}$ avec une moyenne de $34 \pm 5 \mu \mathrm{S} / \mathrm{Cm}$ (figure $2 \mathrm{C}$ et 2D). Les eaux d'Akono correspondent donc à la classe des eaux faiblement minéralisées. Les pourcentages d'oxygénation dans les milieux aquatiques sont restées faibles à létales pendant toute la période d'étude. Elles sont comprises entre 3,3 et $25,2 \%$, avec une moyenne de $13,1 \pm 7 \%$. Ces valeurs ne montrent aucune différence entre les stations. L'oxydabilité à des valeurs comprise entre 0,6 et 3,7 mg/L, avec une moyenne de 1,8 $\pm 0,1 \mathrm{mg} / \mathrm{L}$. Les eaux sont donc faiblement chargées en matière organique. Les teneurs en nitrites sont presque nulles et sont comprises entre de 0 et $0,08 \mathrm{mg} / \mathrm{L}$. De même, les teneurs en azote ammoniacal varient entre 0,1 et $1,05 \mathrm{mg} / \mathrm{L}$ et lesteneurs en nitrates sont comprises entre 0,3 et $4,7 \mathrm{mg} / \mathrm{L}$. Ces teneurs sont inférieures aux normes de l'OMS. Les teneurs des eaux en orthophosphate varient entre 0,7 et $3,5 \mathrm{mg} / \mathrm{L}$ et ne présentent pas de différence entre les stations. 

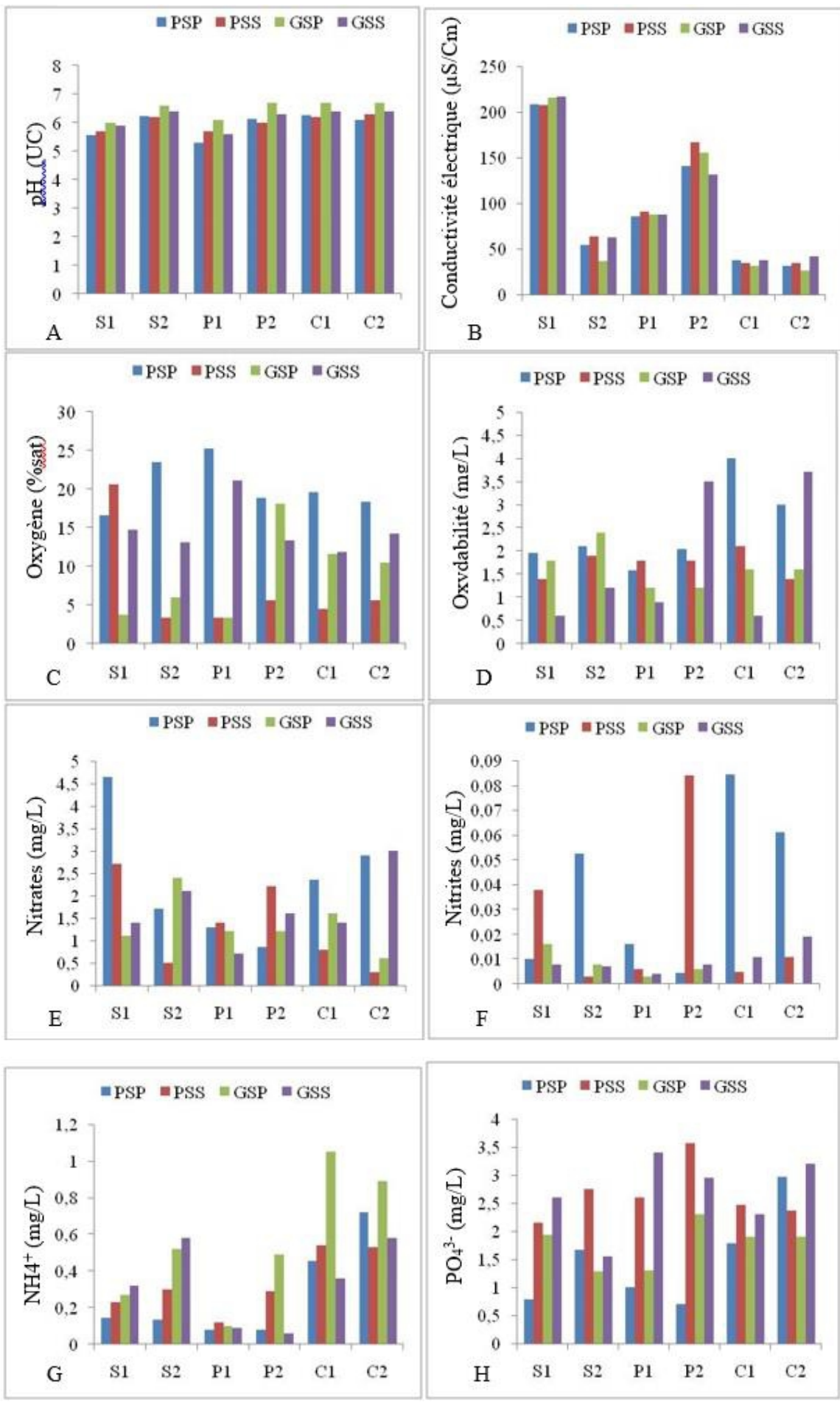

Figure 2 : variations spatio-temporelles des paramètres chimiques dans les sources, puits et cours d'eau de la commune d'Akono : (A) pH, (B) conductivité électrique et (C) oxygène, (D) oxydabilité, $(\mathrm{E})$ nitrates, $(\mathrm{F})$ Nitrites, $(\mathrm{G})$ azote ammoniacal et $(\mathrm{H})$ orthophosphates. 


\subsection{Variables biologique}

\subsubsection{Identification et caractérisation des formes parasitaires-}

$\mathrm{Au}$ total, trois genres de coccidies enteropathogènes ont été identifiés à savoir Crytosporidium sp, Cyclospora cayetanensis et Isospora belli.

Les oocystes de Cyclosporacayetanansis observésont un diamètre compris entre 8 et $10 \mu \mathrm{m}$ et sont de forme sphérique avec une couche fibrillaire externe $(63 \mathrm{~nm})$, qui recouvre une paroi cellulaire épaisse $(50 \mathrm{~nm})$. Les formes non sporulées se présentent avec des grappes de globules de $2 \mu \mathrm{m}$ réfrangibles et les oocystes sporulés présententun sporocystes ou deux sporocystes contenant chacun deux sporozoïtes.
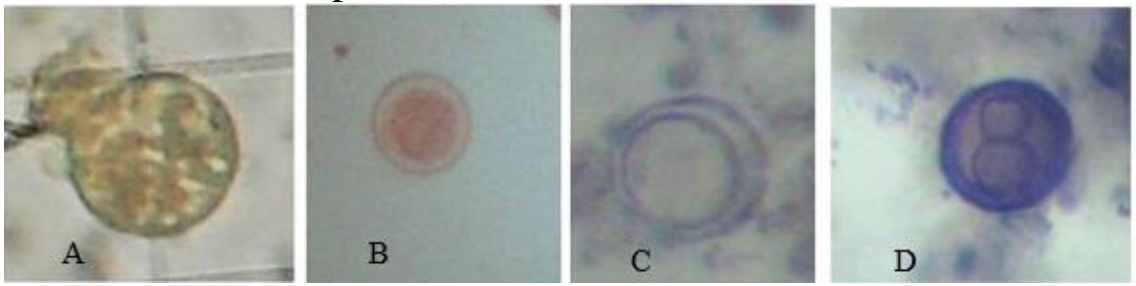

Figure 3 : Oocystes de Cyclospora cayetanensis vus au microscope optique objectif 40X :

$(\mathrm{A}, \mathrm{B})$ Oocystes non sporulés $(\mathrm{C}$ et $\mathrm{D})$ Oocystes sporulés

Les oocystes d'isospora belli quant à eux ont une forme ovalaire, réfringents, avec une double paroi et mesurent de 20 à $30 \mu \mathrm{m}$ de long sur 12 à $16 \mu \mathrm{m}$ de large. Ils contiennent un sporoblaste et parfois deux sporocystes plus ou moins différenciés.
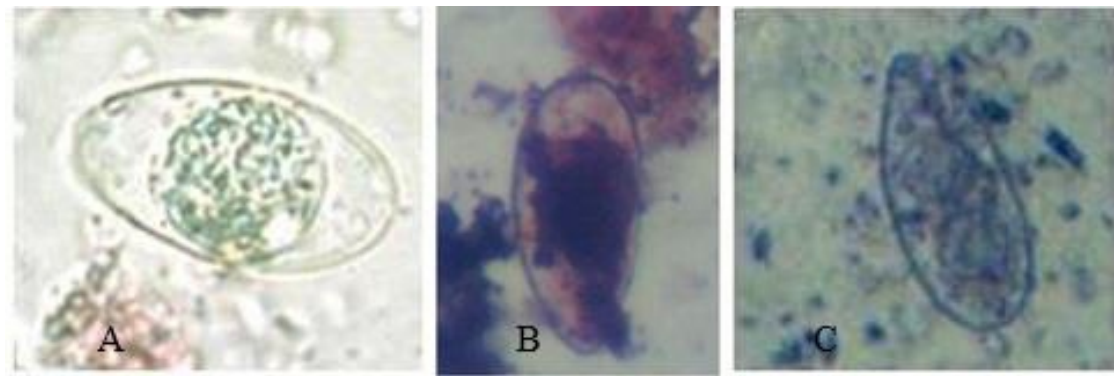

Figure 4: Oocystes d'isospora belli vus au microscope optique : (A et B) Isospora belli non sporulé et sporulé (C)

La figure 5 présente les oocystes de Cryptosporidium sp observés. Ils ont une forme sphérique. Leur diamètre varie entre 4 et $6 \mu \mathrm{m}$ selon les espèces. Leur paroi est épaisse et composée de deux couches, interne et externe, bien distinctes. Leurs contenus est granuleux, aspect réfringent, présentant un point noir bien visible correspondant au corps résiduel. 

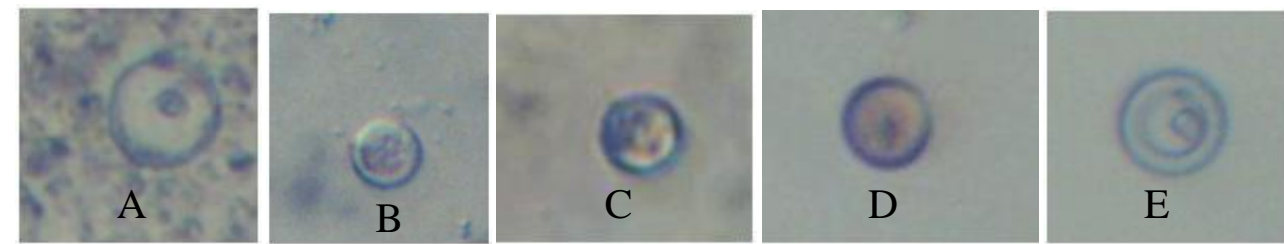

Figure 5 : Oocystes de Cryptosporidium $s p$ (A, B, C, D, E) vus au microscope optique objectif 40X.

\subsection{Caractérisations parasitologiques des sources, puits et cours d'eau échantillonnés}

Les analyses parasitologiques des échantillons d'eau ont permis de mettre en évidence trois espèces de coccidies intestinales dans les sources, les puits et le cours d'eau étudiés. Il s'agit de Cryptosporidium sp, de Cyclospora cayetanensis et d'Isospora belli.

La représentation en diagramme circulaire de la charge parasitaire des trois plan d'eau montre que le cours d'eau est plus chargé en parasite (54\%), suivie des sources (34\%) et des puits (12\%).

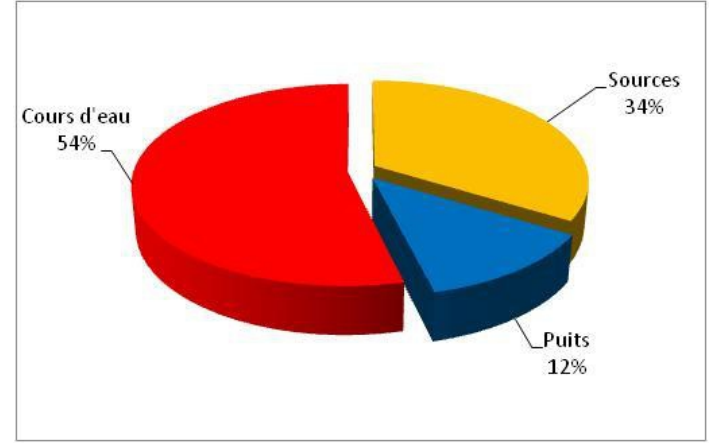

Figure 6 : Représentation de la charge parasitaire dans les milieux aquatiqu

\subsubsection{Variations des densités moyennes des oocystes de Cryptosporidium sp, Cyclospora cayetanensis et Isopora belli dans les milieux aquatiques de la commune d'Akono.}

Les résultats des analyses parasitologiques présentées sur la figure 7 , montrent que les densités moyennes des oocystes dans les milieux aquatiques varient entre 0 et 52 oocystes/L pour toutes les espèces identifiées. Dans les sources et les puits, cette étude montre une abondance totale de 108 oocystes/L, avec une densité moyenne de 79 oocystes/L dans les sources et de 29 oocystes/L dans les puits. La source S2 est la plus contaminé (35 oocystes/L) et le puits P1 (11 oocystes/L) est le moins chargé en parasites (figure 7A). Au niveau du cours d'eau, cette étude a révélé une abondance de 126 oocystes/L. La station C2 du cours d'eau est la plus contaminée (96 oocystes/L) (fig. 7A). Dans le milieu souterrain, les oocystes du genre Cryptosporidium sp ont été les plus abondants (70 oocystes/L), suivie de ceux 
de Cyclospora cayetanensis (28 oocystes/L) et d'Isospora belli (9 oocystes/L) (figure 7A). De même, dans le cours d'eau les oocystes de Cryptosporidium sp ont été les plus abondants (71 oocystes/L), suivie de ceux d'Isospora belli (31 oocystes/L) et de Cyclospora cayetanensis (24 oocystes/L) (figure 8A).

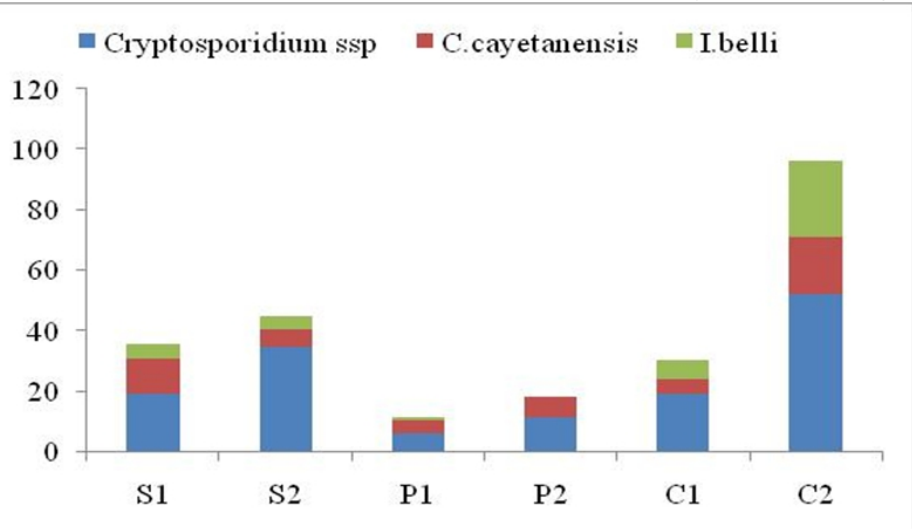

Figure 7: Distribution spatiale des densités moyennes des oocystes identifiés : (A) dans les eaux de sources et des puits et dans le cours d'eau

La figure 8 présente les variations spatio-temporelles des abondances des oocystes des différentes espèces. Cette étude montre que les puits et les sources se caractérisent par une abondance plus élevée en oocystes pendant la PSS. Les sources présentent une abondance plus élevée en PSP. Les abondances les plus faibles sont enregistrées pendant la GSP.

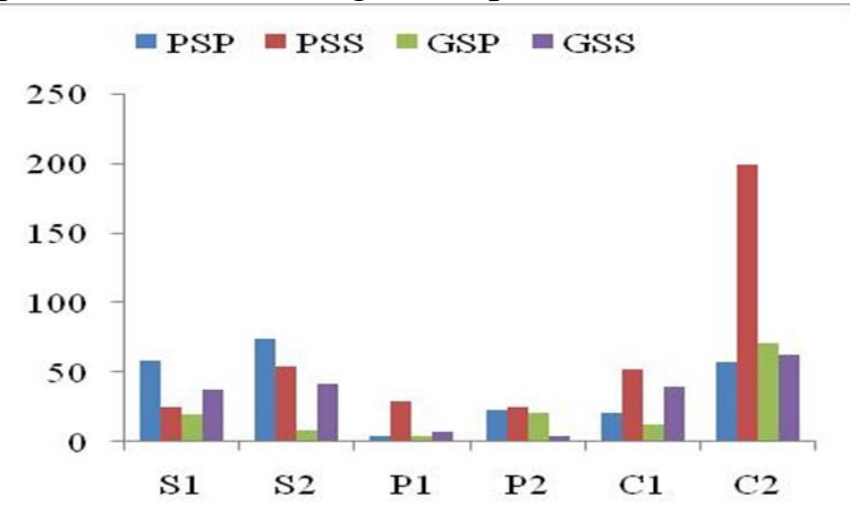

Figure 8: Distribution saisonnière des abondances relatives des oocystes identifiés dans les eaux de sources, des puits et dans le cours d'eau

Tableau II : corrélation entre variables physicochimiques et biologiques.* = corrélation significative au seuil $5 \%$ et $* *=$ corrélation significative au seuil $1 \%$

\begin{tabular}{|c|c|c|c|c|c|}
\hline Paramètres & $\begin{array}{c}\text { Conductivi } \\
\text { té }(\mu \mathrm{S} / \mathrm{cm})\end{array}$ & $\begin{array}{c}\text { TDS } \\
(\mathrm{mg} / \mathrm{L})\end{array}$ & MES $(\mathrm{mg} / \mathrm{L})$ & $\begin{array}{c}\text { Couleur } \\
\mathrm{pt} / \mathrm{co}\end{array}$ & $\begin{array}{c}\text { Turbidité } \\
(\mathrm{FTU})\end{array}$ \\
\hline Cryptosporidium sp & $-0,371^{* *}$ & $-0,354^{* *}$ & $0,436^{* *}$ & $0,480^{* *}$ & $0,418^{* *}$ \\
\hline Cyclospora $\mathrm{spp}$ & $-0,32$ & $-0,008$ & $0,285^{*}$ & 0,198 & 0,191 \\
\hline
\end{tabular}




\begin{tabular}{|c|c|c|c|c|c|}
\hline & & & & & \\
\hline Isospora spp & $-0,90$ & $-0,062$ & 0,129 & 0,120 & 0,103 \\
\hline
\end{tabular}

La densité des oocystes de Cryptosporidium sp a été négativement et significativement corrélé à la conductivité $(\mathrm{r}=-0,371 ; \mathrm{p}<0,01)$ et positivement corrélé aux variables suivante : MES $(r=0,436 ; p>0,05)$, couleur $(r=0,480$; $p>0,01)$ et la turbidité $(r=0,418 ; p>0,01)$. Ces résultats révèlent que la densité des oocystes identifiés décroit lorsque le niveau de minéralisation des eaux augmente. La densité des oocystes de Cryptosporidium sp, C.cayetanensis, Isospora belli croit lorsque les MES, la couleur et la turbidité de l'eau augmente.

TableauIII. Comparaison des Moyennes des paramètres physico-chimiques avec les normes (OMS, 2010)

\begin{tabular}{|l|l|l|l|l|l|}
\hline paramètres & Unité & Normes OMS & Minimum & Maximum & Moyenne \\
\hline $\mathrm{Ph}$ & $\mathrm{UC}$ & $6,5-8,5$ & 5,3 & 6,7 & $6,1 \pm 0,3$ \\
\hline $\mathrm{T}$ & ${ }^{\circ} \mathrm{C}$ & - & 23,8 & 26 & $23,8 \pm 2$ \\
\hline $\mathrm{CE}$ & $\mu \mathrm{S} / \mathrm{Cm}$ & 250 & 34 & 218 & $95,5 \pm 67,5$ \\
\hline $\mathrm{NO} 3-$ & $\mathrm{mg} / \mathrm{L}$ & 50 & 0,6 & 3,7 & $1,6 \pm 0,9$ \\
\hline $\mathrm{NO} 2-$ & $\mathrm{mg} / \mathrm{L}$ & 3 & 0 & 0,08 & $0,01 \pm 0,02$ \\
\hline $\mathrm{PO} 4-$ & $\mathrm{mg} / \mathrm{L}$ & - & 0,7 & 3,5 & $2,1 \pm 0,7$ \\
\hline $\mathrm{NH} 4+$ & $\mathrm{mg} / \mathrm{L}$ & $0,2-0,3$ & 0,06 & 1,05 & $0,3 \pm 0,2$ \\
\hline MES & $\mathrm{mg} / \mathrm{L}$ & 15 & 4 & 24 & $10,7 \pm 5,1$ \\
\hline Couleur & $\mathrm{Pt}-\mathrm{Co}$ & 15 & 22,2 & 267 & $99,6 \pm 76,2$ \\
\hline turbidité & $\mathrm{FTU}$ & $>5$ & 2 & 43 & $10,2 \pm 9,5$ \\
\hline
\end{tabular}

\section{Discussion}

La faible minéralisation des eaux obtenue est contraire aux résultats obtenus dans les eaux de sources, puits et cours d'eau de la localité de Yaoundé (Kapso, 2019) mais proche des données d'Ajeagha et al (2017). Cette différence pourrait s'expliquer par la faible dégradation de la matière organique présente dans les milieux aquatiques et traduirait le caractère peu pollué de ces eaux par rapport à celles de la capitale Yaoundé où la pollution est plus importante.

Le $\mathrm{pH}$ de l'eau est légèrement acide avec des valeurs comprises entre

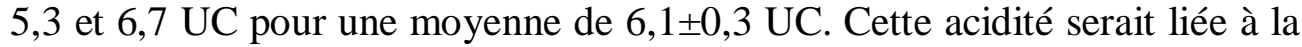
nature argileuse et ferralitique des sols de la localité. En effet, le pH des eaux naturelles est lié à la nature des terrains traversés (Jullian et al., 2005). Dans les milieux aquatiques les sources d'azote sont d'origine anthropique et comprennent l'azote du sol, les engrais, les eaux usées, les fosses septiques, les déchets d'animaux et les résidus de plantes. Les fortes teneur en azote dans l'eau sont toxiques et provoquent la méthémoglobinémie chez les enfants. Toutefois dans la présente étude, les teneurs en azote sont restées inférieurs aux normes.

La turbidité neutralise l'action des désinfectants. Selon LeChevallier et Norton (2008), le pouvoir désinfectant du chlore diminue de huit fois 
lorsque la turbidité passe de 1 à 10. Les valeurs de la turbidité, de la couleur et des MES obtenues dans cette étude sont dans l'ensemble supérieurs aux normes et témoignent d'un enrichissement modéré en matière organique de ces eaux.

Les analyses parasitologiques des échantillons d'eau de la commune d'Akono révèlent la présence des formes de résistances des coccidies entériques dans les sources, les puits et le cours d'eau. La présence des microorganismes dans les puits et les sources étudiés pourrait s'expliquer par la faible profondeur de la majorité des puits et des sources ainsi que la proximité avec les latrines. Par ailleurs, la non-étanchéité et le mauvais entretient ont pu également favoriser la contamination par les protozoaires entériques liée à l'infiltration des eaux usées (Hynds et al., 2014). Les faibles densités de parasites enregistrées dans les eaux souterraines par rapport aux eaux de surface peuvent s'expliquer par le fait que les eaux souterraines subissent une filtration. Cette filtration s'effectue de façon naturelle par le sol et limite la contamination de la nappe. De plus, ces oocystes peuvent survivre à des températures aussi élevées pendant une période de six mois (ANSES, 2011).

Les paramètres des eaux naturelles peuvent influer sur la qualité biologique de l'eau. Les corrélations positives observées entre les MES, la Couleur et la Turbidité confirment ce fait. En effet, plusieurs études ont établi l'existence de corrélations entre l'augmentation des concentrations de microorganismes et une turbidité accrue (Snead et al., 2011; Goshko et al., 2011; Haas et al., 2011). La réduction de la turbidité permet l'inactivation des microorganismes surtout les oocystes de-Cryptosporidium. L'homme se contamine en consommant de l'eau non traitée ou en ingérant des aliments contaminés. Après ingestion par l'homme, les oocystes perdent leur coque protectrice dans le tube digestif et libèrent des sporozoïtes, qui envahissent les cellules épithéliales de l'intestin grêle et causent de la diarrhée, des crampes abdominales ou des ballonnements et des nausées (Ortega et al., 2010). La qualité de l'eau et de la nourriture devrait donc être une priorité.

\section{Conclusion}

Cette étude révèle que les eaux de sources, de puits et du cours d'eau échantillonnées dans la commune d'Akono ont été de qualité physicochimique moyenne avec une faible pollution organique et une faible minéralisation. En ce qui concerne la qualité biologique, les eaux de la commune d'Akono ont renfermé des formes de résistances de coccidies intestinales. Les eaux de puits ont contenu moins d'oocystes que les eaux de sources et de cours d'eau. Les oocystes de cryptospridium ont été les plus abondants suivis de ceux de Cyclospora cayetanensis. La densité des oocystes a été corrélée positivement aux teneurs en MES, à la couleur et à la turbidité. 
Cette étude a montrée que l'utilisation de ces eaux par les sans traitement préalable pour des besoins domestique, de nutrition et de récréation peut entrainer à long terme des risques sanitaires. Nous recommandons aux pouvoirs publics d'étendre le réseau de distribution d'eau potable au niveau des zones rurales. Aux riverains de procéder à la désinfection de l'eau par ébullition.

\section{References}

1. ANSES (Agence National de Sécurité Sanitaire, alimentation, environnement et travail). (2014) fiche de description de danger biologique transmissible par les aliments.

2. Ajeagah, G.A., Njine, T., Bilong Bilong, C.F., Foto, S.M., Wouafo, N.M., Nola M., DiG.G.D. et Huw, S. (2010). Seasonal Distribution of Enteric Opportunistic Cryptosporidium Spp. oocysts and Giardia Spp. Cysts in a tropical water basin, Cameroon. Department of Parasitology, Faculty of Public Health, Mahidol university, Thailand : 44-57.

3. Ajeagah, G.A. (2017). Water as a weapon of international confrontations. Harmattan ; Cameroun Environnement, Nature, Écologie-Géographie Afrique Subsaharienne Cameroun, 220p.

4. Allan, J. D. (2004). Landscape and rivers cape: the influence of land use on stream ecosystems.

5. Annals Review of Ecological Systems, 35: 257-284.

6. American Public Health Agency. (Ed).(1998). Standard methods for examination of water and waste water. A.P.H.A. - AWWA-WPCF, Pennsylvania, Washington DC., $1150 \mathrm{p}$.

7. De Villers, J., Squilbin, M., et Yourassowsky, C. (2005). Qualité physicochimique et chimique des eaux de surface. Institut Bruxellois pour la gestion de l'environnement.

8. Goshko, M.S., Minnigh, H.A., Pipes, W.O. et Christian, R.R. (2011). Relationships between standard plate counts and other parameters in water distribution systems. J. Am. Water Works Assoc., 75(11): 568571

9. Haas, C.N., Meyer, M.A. et Paller, M.S. (2011). Microbial alterations in water distribution systems and their relationship to physicalchemical characteristics. J. Am. Water Works Assoc., 75: 475-481. Review.

10. Hynds, P.D., Thomas, M.K. et Pintar, K.D.M. (2014). Contamination of groundwater systems in the US and Canada by enteric pathogens, 1990-2013: a review and pooled-analysis. PLOSOne, 9(5): e93301.

11. INC. (1980).Carte topographique de Yaoundé et ses environs au 1/500, Yaoundé : Institut Nationale de Cartographie. 
12. INS (Institut national de Statistique). (2013). Statistique annuelle, Yaoundé-Cameroun.

13. Jullian, E., Hirbec, A., Ker, B.N. et Liu, R. Z. (2005). 'Qualité de l'eau du basin versant de l'Ardèche." Université de Paris 7 - Denis - Diderot UFR des sciences physiques de la terre (IUP Génie de l'Environnement).

14. Kapso, T. (2018). Dynamique d'abondance des amoebidés dans les milieux aquatiques à Yaoundé (Cameroun). Thèse de Doctorat/PhD, Faculté des Sciences. Université de Yaoundé ,I, 213 p.

15. LeChevallier, M.W. et Norton, W.D. (2008). Examining relationships between particle counts and Giardia, Cryptosporidium, and turbidity. J. Am. Water Works Assoc., 84(12):54-60.

16. Ortega, YR., Sanchez, R. (2010 jan). Update on Cyclospora cayetanensis, a food-borne and waterborne parasite. Clin Microbiol Rev; 23(1):218-34.

17. Rodier, J. (2009). L'analyse de 1'eau. 9e édition, Dunod, Paris, 1526p.

18. Santos, S.F.O., Silva, H.D., Souza, E.S.J., Anunciaçăo, C.E., LacerdaSilveira, P.E., Vilanova-Costa, C.A.S.T. et Garciazapata, M.T.A. (2010). Environnemental monitoring of opportunistic protozoa in rivers and lakes in the neotropics based on yearly monitoring. Water Quality of Exposition Health, 2: 97-104.

19. Snead, M.C., Olivieri, V.P., Kruse, C.W. et Kawata K. (2011). Benefits of maintaining a chlorine residual in water supply systems. U.S. Environmental Protection Agency, Washington, DC. Juin (EPA600/2-80-010).

20. TUEKAM, K.R.P. (2013). Essai de biotypologie faunistique des eaux souterraines dequelques localités des régions du Centre et Littoral du Cameroun: influence de quelquesfacteurs abiotiques. Thèse de Doctorat/PhD, Faculté des Sciences. Université de YaoundéI, 291 p.

21. UNESCO. (2003).- Faits et Chiffres : Approvisionnement en eau et assainissement. http :// www.unesco.org/année internationale de l'eau douce.html. [Consulté le 5 juin 2019].

22. Vilagines, R. (2003). Eau, environnement et santé publique. Lavoisier(Éditeur), Paris

23. Zébazé Togouet, S.H., Tuekam Kayo, R.P., Boutin, C., Nola, M. et Foto Menbohan, S. (2011). Impact de la pression anthropique sur l'eau et la faune aquatique des puits et sources de la région de Yaoundé (Cameroun, Afrique Centrale). Bulletin de lasociété d'Histoire Naturelle de Toulouse, 147: 27-41 